\title{
Plant-Microbe Interactions and Water Management in Arid and Saline Soils
}

Daniele Daffonchio ${ }^{1,2} *{\text { Heribert } \text { Hirt }^{2} \text { and Gabriele Berg }}^{3}$

${ }^{1}$ DeFENS, Department of Food, Environmental and Nutritional Sciences, University of Milan, I-20133 Milan, Italy

${ }^{2}$ King Abdullah University of Science and Technology, BESE Division, Thuwal, 239556900, Kingdom of Saudi Arabia

${ }^{3}$ Institute of Environmental Biotechnology, Graz University of Technology, A-8010 Graz, Austria

*Email: daniele.daffonchio@unimi.it

\begin{abstract}
Drought and salinity are major factors limiting agriculture in many regions in the world, and their importance is predicted to even increase in the near future in parallel with the ongoing global warming and climate changes. Soil and rhizosphere microbes are potential resources for counteracting such abiotic stresses in plants. The knowledge on the roles of root microorganisms in retaining soil humidity and promoting plant growth under such abiotic stresses is analyzed in this chapter. The importance of microbial diversity in the rhizosphere for alleviating drought and salinity effects on the plant physiology is discussed in the light of "Desert Farming", the general crop management practice that is frequently used in arid regions. The plant growth promoting functional services exerted by microorganisms within the rhizosphere in arid soils are presented in relation to the plant response under water stress.
\end{abstract}

\subsection{The Effects of Drought and Salinity on Plant Physiology}

A major challenge for future agriculture is to cope with the increasing demand for food production, facing a constantly increasing world population. This growing demand for agricultural production is paralleled by dramatic losses of arable land due to enhanced soil destruction and erosion. Drought and soil salinity are the two major environmental factors that limit plant growth and development thereby negatively affecting agricultural yield by more than $60 \%$. Water shortage is critical in many areas of the world and is usually countered by extensive irrigation. Although the planet earth is rich in water, not only the water of the oceans, but also most inland water resources are highly saline. Moreover, irrigation usually results in soil salinization making drought and soil salinity increasing agricultural problems (Bartels and Sunkar 2005).

To convert deserts into arable, green landscape is a global vision as well as competent answer to world hunger and climate change (Clery 2011). Desert farming, which generally relies on irrigation, is part of this vision. Agriculture systems were already developed in arid 
landscapes by ancient cultures, yet nowadays, there is a dramatically increasing need for large-scale desert farming to feed the population. Desert farming is not only a challenge for irrigation systems with impact on the global water balance, it can also have an impact on soil microbial diversity. Changes of soil bacterial diversity, especially reduced beta diversity, was shown to occur in a semi-arid ecosystem as a consequence of land use for agriculture with potential irreversible consequence on the natural soil ecosystem (Ding et al. 2013). To avoid this risk and to establish sustainable desert farming systems it is important to understand diversity in arid and saline environments.

Salt and drought stress share some properties and generally result in impaired key physiological functions. One component of salinity is hyperosmotic stress resulting in a water deficit that is comparable to a drought-induced water deficit. The other component of salt stress is ion toxicity, resulting in metabolic imbalance. Membranes may become disorganized, proteins may become inactive and excess levels of reactive oxygen species (ROS) can be produced leading to oxidative damage. As a consequence, inhibition of photosynthesis, metabolic dysfunction, and damage of cellular structures contribute to plant growth inhibition, reduced fertility and premature senescence.

Plants basically counteract the negative effects of salinity and drought by activation of genetic and biochemical responses. These responses include the synthesis and accumulation of osmolytes, such as proline or raffinose, that are able to stabilize proteins and cellular structures but also to maintain cell turgor pressure by osmotic adjustment. Moreover, plants also enhance scavenging of ROS, which are generated as a secondary effect of salt and drought stress. Several plant stress signaling pathways have been dissected in detail in the model organisms Arabidopsis thaliana and rice and a number of central transcription factors have emerged that regulate cohorts of downstream genes. Despite this tremendous progress, our knowledge on the coordination and integration of these regulatory pathways into the complex matrix of plant stress physiology is still limited. Moreover, different plant species developed different strategies to cope with stress. For example, some halophytic Brassicacae, such as the Arabidopsis-related halophyte Thellungiella halophyla, avoid stress by salt exclusion, whereas Lobularia maritima accumulates and detoxifies salt by compartmentalisation. Moreover, harsh environmental conditions, which are harmful for one plant species, might not be stressful for another species. These differences correlate with different stress-response mechanisms and two main strategies of stress avoidance and stress tolerance can be found in the plant kingdom. Stress avoidance in some species is a genetically inherited trait that delays or prevents the negative impacts of a stress. For example, cacti show a permanently adapted morphology and physiology to hot and arid climates. Stress tolerance is an adaptive strategy to counterbalance stress conditions and most plants can adapt to drought conditions by closing their stomates to reduce transpirational water loss. Most plants can also acclimate to stress conditions upon a gradual and repeated increase of a stress factor. Acclimation induces various physiological changes that are reversed when the adverse environmental conditions disappear. Overall, whereas the avoidance mechanisms are usually constitutive features that are genetically inherited, acclimation mechanisms are plastic and reversible. At the molecular level, acclimation involves the modification of gene expression 
but also epigenetic mechanisms. Stress-inducible genes comprise genes involved in direct protection from stress, including the synthesis of osmoprotectants, detoxifying enzymes, and transporters, as well as genes that encode regulatory proteins such as transcription factors, protein kinases, and phosphatases.

An issue that is worth to be explored for the future of agriculture is the effects that plant associated microorganisms may exert on all these metabolic processes for improving plant resistance and resilience to drought and salinity stresses.

\subsection{Rhizosphere Bacterial Diversity in Arid and Saline Soils and under "Desert Farming"}

Arid and Saline Soil Habitats Harbour a Unique Bacterial Diversity. Deserts represent in general extreme environments for microorganisms. Although the conditions varied strongly in the different regions of the world, all of them are characterized by a combination of extreme temperatures and desiccation, resulting in arid and saline soils. These extreme abiotic factors, as well as the absence of plants at many sites, contribute to the visual appearance of a sterile environment. While early studies supported this "sterility" by very low levels of viable/cultivable microorganisms, applications of DNA/RNA-based molecular methods led to interesting new insights and showed a contrasting picture. For example, in their global-scale study, Fierer and Jackson (2006) found that the acidic soils of tropical forests harbour fewer bacterial taxa than the neutral $\mathrm{pH}$ soils of deserts. In different sites in the Negev Desert, archaeal and bacterial diversity was not constrained by precipitation, although the taxonomic composition differed (Angel et al. 2010). In soil of the Atacama Desert, a high diversity of microorganisms known to live in hypersaline environments was found by analysis of Denaturing Gradient Gel Electrophoresis profiles of amplified 16S rRNA gene (de Los Ríos et al. 2011). Most of the desert microbial communities seem to be structured solely by abiotic processes. However, if desert plants are present, they strongly shape soil microbial diversity. Also desert animals play an important role as ecosystem engineers and affect the microbial complex of diazotrophs in soils. All these investigations showed a unique and extraordinary microbial diversity in belowground desert environments, strongly adapted to their unique conditions.

The SEKEM Farms as Example for Sustainable Desert Farming. On the SEKEM farms in Egypt, desert land was converted into arable land, and biodynamic agriculture is operated for over 30 years now (www.sekem.com). Today SEKEM is carrying out organic agriculture on more than 4,100 hectares and has the largest market for organic products outside Europe and North America. They produce organic foods, spices, tea, cotton textiles and natural remedies. However, the cultivation especially of medical plants is more and more affected by soil-borne phytopathogens, which lead to significant yield losses. Therefore, this is an excellent study example i) to understand the impact of desert farming on microbial diversity and, ii) to develop a specific biocontrol strategy for desert agriculture. 
To examine the impact of organic agriculture on bacterial diversity and community compositions in desert soil, soil from a SEKEM farm in comparison to the surrounding desert soil from different sites were assessed by pyrosequencing-based analyses of 16S rRNA and nifH gene sequences (Köberl et al. 2011). In addition, fingerprinting and cultivationdependent methods were included in this study. Altogether, a strong impact on the structure and function of the bacterial and fungal communities was found. The computed Shannon indices of diversity ( $\mathrm{H}^{\prime}$ ) calculated for bacterial communities on the basis of amplicon libraries were much higher for agricultural soil than for desert soil ( $\mathrm{H}^{\prime}$ at a dissimilarity level of 20\%: SEKEM soil: 4.29; desert soil: 3.54 ). This indicates a higher bacterial diversity in soil due the agricultural use of the desert. For both soil types together 18 different phyla were identified; dominant groups present in both soils were especially Proteobacteria $(30.2 \%)$, Firmicutes (27.3\%) and Actinobacteria (10.5\%). In detail, Firmicutes were highly enriched in agricultural soil (from $11.3 \%$ in desert soil to $36.6 \%$ in SEKEM soil), while Proteobacteria (46.0\% in desert soil and $21.0 \%$ in SEKEM soil) and Actinobacteria (20.7\% in desert soil and $4.6 \%$ in SEKEM soil) occurred in SEKEM in lower abundances than in the surrounding desert. In addition, in both soils Bacteroidetes (4.6\% and 5.3\%) and Gemmatimonadetes $(1.4 \%$ and $1.9 \%)$ were present. Whereas Acidobacteria $(7.9 \%)$ and Planctomycetes $(1.1 \%)$ were only present in the agricultural soil, Deinococcus-Thermus (1.1\%) was only detectable in the desert sand. In contrast to other studies, the most important difference was the high abundance of Firmicutes. Most of the Firmicutes were classified as Bacillus; in the agricultural soil also the phylogenically related genus Paenibacillus was found $5 \%$ of classified Firmicutes). These differences between the two soil types found for the bacterial community could be confirmed for the fungal community analyzed by fingerprinting and for the functional studies regarding antagonistic microorganisms and diazotrophs (Köberl et al. 2013a; Köberl et al. 2013b). After long-term farming, a drastic shift in the microbiomes in desert soil was observed. Bacterial communities in agricultural soil showed a higher diversity and a better ecosystem function for plant health but a loss of extremophilic bacteria. Interestingly, we detected that indigenous desert microbes promoted plant health in desert agro-ecosystems.

In desert soils, plant roots provide important nutrient sources for bacteria and act as microbial hot spot. The composition of the microbial community in rhizosphere and endorhiza of three different medical plants (Matricaria chamomilla L., Calendula officinalis L. and Solanum distichum Schumach. \& Thonn.) grown under organic conditions on SEKEM farms confirmed this hypothesis. These results show that dominant bacteria, e.g. Ochrobactrum and Rhodococcus, are taken up by the plants from the soil and that soil is the main reservoir for plant-associated bacteria. Further, nearly in all samples Bacillus sp. was found. The fungal community fingerprints included a quite high diversity in all microenvironments. Verticillium dahliae, a prominent phytopathogenic fungus, was found nearly in all samples on the SEKEM farms. In general, mainly potential plant pathogens were found within the fungal communities. The obligate root-infecting pathogen Olpidium, (fungal phylum Chytridiomycota), was found especially in the rhizosphere and endorhiza of M. chamomilla. Alternaria and Acremonium were primarily present in the rhizosphere samples. There were significant differences between the rhizosphere and the endorhiza of the medical plants. In 
general, samples from the rhizosphere generated more strains than samples from the endorhiza of the medical plants, which indicate that a sub-set of rhizobacteria was able to invade the root. Diazotrophs are key organisms for providing nitrogen in natural or organically managed agricultural ecosystems, especially under desert conditions. By combining nifH-specific quantitative PCR, fingerprints, amplicon pyrosequencing and fluorescent in situ hybridization-confocal laser scanning microscopy, a generally high nifH abundance and diversity in native and agricultural desert soil and in the rhizosphere of medicinal plants was detected, the highest reported until now compared with other ecosystems. Statistically significant differences were found between both soil types (native and agriculturally used), between the different microhabitats (bulk soil, rhizosphere, endorhiza), and between the three investigated medicinal plants. Again, a considerable community shift from desert to agriculturally used soil was observed with higher abundance and diversity in the agro-ecosystem. In comparison to the rhizosphere, the endorhiza was characterized by lower abundances and a subset of species. Comparing root-associated communities, remarkable differences were found. While the microbiomes of $M$. chamomilla and $C$. officinalis were similar and dominated by potential root-nodulating rhizobia mainly acquired from soil, the perennial $S$. distichum formed primarily associations with free-living nitrogen fixers most likely transmitted between plants, possibly by mean of the seeds, as they are undetectable in soils. The results underline the importance of diazotrophs in desert ecosystems and moreover identified plants as important drivers for functional diversity.

The major problems in the cultivation of plants on SEKEM farms are caused by the soilborne pathogenic fungi Verticillium dahliae Kleb., Rhizoctonia solani J.G. Kühn and Fusarium culmorum (Wm.G. Sm.) Sacc. as well as by the soil-borne pathogenic bacterium Ralstonia solanacearum. Bacterial isolates obtained from the soil of the SEKEM farm exhibited a higher in vitro antagonistic potential towards soil-borne phytopathogenic fungi in comparison to the bacteria isolated from the desert soil (SEKEM $21.2 \pm 1.2 \%$; desert $12.6 \pm$ $0.8 \%$ ). From the agricultural soil $17.4 \%$ (27 isolates) demonstrated antagonism towards all three fungal pathogens, while only 10.6\% (21 isolates) from the desert soil. Already the desert soil harbors a high proportion of antagonists, which were augmented by organic agriculture in SEKEM soil. The soil from the farm seems to be supplied with antagonists in such an optimal way, that there was no detectable enrichment of antagonists in the rhizosphere and endorhiza of the investigated medical plants. In general, M. chamomilla and S. distichum showed a better antagonistic potential than $C$. officinalis. Especially the endorhiza from M. chamomilla harbors a high proportion of antagonists. Whereas most antagonistic bacteria against Fusarium culmorum were found in the soil and in the rhizosphere of the medical plants, those against Verticillium dahliae were found in the endorhiza. A representative selection of promising biological control agents was identified by partial 16S rRNA gene sequencing as members of the Bacillus subtilis and Bacillus cereus groups, and of the genera Paenibacillus, Streptomyces and Lysobacter. Except for one isolate of Lysobacter, only gram-positive antagonists were found. All microenvironments were dominated by antagonists from Firmicutes with Bacillus and Paenibacillus isolated from all habitats. Antagonistic Streptomyces were found exclusively in desert soil. Promising strains of Streptomyces subrutilus, Bacillus subtilis and Paenibacillus polymyxa were tested for their activity under 
field conditions on chamomile seedlings. The Bacillus and Paenibacillus strains enhanced the production of the bioactive secondary plant metabolite apigenin-7-O-glucoside (Schmidt et al. 2013). Other Bacillus subtilis isolates were able to control root-knot nematodes by inducing systemic resistance of tomato plants (Adam et al. 2014).

\subsection{Functional Services of Rhizosphere Bacteria under "Desert Farming"}

The wide diversity of bacteria in the rhizosphere is exploited in a series of services that help plants in counteracting drought and salinity stresses. Such services, ranging from general ecological services to contributions to the physical protection of the root from the mechanical stress or in the plant hormone homeostasis, are important features under Desert Farming.

Microbial Ecological Services. The ecology of the arid systems modulates the type of microorganisms and the extent of the services provided to plants. For instance the potential plant growth promoting (PGP) services provided by the root associated bacteria appear to be invariant respect to macroecological factors such as latitude, soil type and plant cultivar, despite provided by different bacterial communities, according to observations across an aridity macrotransect from North Italy to North Tunisia and Egypt (Marasco et al. 2013a). Rhizosphere and endophytic bacteria are capable of promoting the growth of plants challenged by drought. It has been shown that such a trait is not a per se capability of the selected microorganisms but it is associated to the water stress (Rolli et al. 2014). So, rootassociated bacteria that normally do not present in-vivo promotion activities can determine plant resistance to drought, suggesting that the services to plant are stress-dependent.

Protection from the Soil Mechanical Stress. Water evaporation and drought determine important changes in the soil aggregates with modifications in the soil particle architecture. Such changes influence the mechanical interactions of the soil with the root surface and the overall physical condition of the soil including water circulation, air exchanges and temperature in the soil. Rhizodeposition determines favorable conditions for bacterial growth. Bacterial biofilms produce exopolymers (EPS) that affect the binding of soil particles to the rhizoplane, improve the water retention in the rhizosphere and protect the root surface from mechanical damages determined by the soil hardness. Plants treated with EPS-producing strains and maintained under drought conditions have increased biomass and cause increased macroporosity in the root adhering soil compared to the non-treated controls.

Protection from Osmotic and Oxidative Stresses. Many bacteria are capable of mitigating water stress in plants by stimulating the production of osmoprotectants in the associated plants. The capacity of both Gram negative (Azospirillum and Pseudomonas) and Gram positive (Bacillus) strains to promote resistance of basil plants to water stress has been associated to increased concentrations of proline and soluble carbohydrates in root and leaf tissues. In the treated plants chlorophyll content increased significantly, confirming the overall beneficial effects of bacteria against drought (Heidari et al. 2011). Bacteria-mediated protection against water stress was also associated to the accumulation of anthocyanin in the vacuoles of mountain laurel (Kalmia latifolia L.), when the stressed plant was colonized by an 
endophytic Streptomyces padanus actinomycete (Hasegawa et al., 2004). Bacteria engineered for overexpressing genes for threhalose synthesis increased their osmotic stress tolerance as well as resistance to drought of their host plants to which they were associated to (RodriguezSalazar et al. 2009).

Cell turgor is an essential process for maintaining normal physiological activities in plant tissues. During drought the aqueous vacuoles in the plant cells regulate the concentrations of osmolytes to decrease the vacuolar osmotic potential and improve water uptake (Park et al. 2005). Different compatible solutes are accumulated in the vacuoles, including sugars, glycine betaine, amino acids, organic acids and pigments. These molecules contribute to protection of the plant from stress through osmotic adjustment, stabilization of the native structure of enzymes and cell membranes and detoxification of ROS induced by the stressful conditions. The increased concentration of such solutes raises the cell's osmotic pressure, contributing to maintain turgor by preventing water loss and promoting water uptake. The overexpression in Arabidopsis as well as in crop plants (such as tomato) of the vacuolar $\mathrm{H}^{+}$-pyrophosphatase, an enzyme implicated in the vacuolar ion turnover, determines improved resistance to drought and enhanced growth compared to the non-engineered controls. The engineered plants have enhanced pyrophosphate-driven cation transport into root vacuoles and increased root biomass and are capable of recovering from exposure to water stress.

The concentration of ROS, such as superoxide, hydrogen peroxide, hydroxyl radical and singlet oxygen, increases in plant tissues under water stress (Apel and Hirt 2004). ROS react with proteins, lipids and nucleic acids, impairing cell physiological functions. In parallel to the promotion of the synthesis of osmolytes in plants exposed to water and salinity stresses, PGP bacteria (PGPB) can enhance tolerance to oxidative stress through the synthesis of stress-related enzymes. For instance, inoculation of drought stressed Lactuca sativa with PGP microbes resulted in increased activity of catalase enzymes (Kohlera et al. 2008).

Effects on Hormone Homeostasis. PGPB are capable of producing plant hormones including auxins, gibberellins (GAs), cytokinins, abscisic acid (ABA) and ethylene (see Chap. 24). Despite the difficulty of separating the effects of bacteria vs. plant-produced hormones, experiments using hormones synthesis inhibitors as well as Arabidopsis mutants impaired in specific hormone synthetic pathways have shown some potential effects of different bacteriaproduced hormones on stimulating resistance to drought and salinity. By using chemical inhibitors of GAs and ABA synthesis in planta, Azospirillum lipoferum treated maize seedlings showed drought stress alleviation, suggesting that bacterial ABA and GAs may play roles in water stress mitigation. Bacterial production of volatile organic compounds (VOCs) (see Chap. 7), such as 2R,3R-butanediol, have been proposed to influence the overall plant hormone balance affecting transpiration and water loss under drought by influencing stomata closure or contributing to shape root architecture potentiating the uptake of water, minerals and microelements in water stressed plants (Cho et al. 2008).

Bacteria can also produce enzymes capable of disrupting plant hormone synthetic pathways, such as the enzyme 1-aminocyclopropane-1-carboxylic acid (ACC) deaminase, which hydrolyzes the plant ethylene precursor ACC to ammonia and $\alpha$-ketobutyrate. Such 
traits may have roles in promoting resistance of plants to drought and salinity. ACC deaminase activity has been associated with stimulation of resistance to salinity stress. For instance, an ACC deaminase-producing Achromobacter piechaudii strain decreased the ethylene production by tomato seedlings grown in the presence of up to $172 \mathrm{mM} \mathrm{NaCl}$, and increased the plant fresh and dry weights (Mayak et al. 2004).

\subsection{The Plant Response to Plant Growth Promoting Bacteria under Stress}

Plants can generate a large portion of their energy by photosynthesis, but plant growth requires significant quantities of nitrate (see Chap. 21), phosphate (see Chap. 22), and other minerals, which are often not freely available in the soil. Root-associated beneficial microbes are important partners of plants and in exchange to carbohydrates, provide many of the limiting minerals. The best-known beneficial microbes are mycorrhizal fungi (see Chap. 23) and rhizobia (see Chap. 21). Mycorrhiza interact with about $80 \%$ of all terrestrial plant species and provide phosphate and nitrate to plants. Free-living or endophytic rhizobia can fix atmospheric nitrogen, but only the family of leguminosae profits from such an interaction through their ability to house rhizobia in root nodules. Although the interaction of plants with mycorrhizal fungi and rhizobial bacteria is well understood, other rhizosphere microbes have received much less attention.

Soil-grown plants are immersed in a sea of microbes and diverse beneficial microorganisms such as PGPB as well as plant-growth promoting fungi (PGPF) can stimulate plant growth and/or confer enhanced resistance to biotic and abiotic stresses (De Zelicourt et al. 2013). The establishment of beneficial plant-microbial interactions requires the mutual recognition and a considerable orchestration of the responses at both the plant and the microbial side. Rhizobial and mycorrhizal symbioses share a common plant signaling pathway that is activated by rhizobial and mycorrhizal factors (Corradi and Bonfante 2012; Geurts et al. 2012) and this signaling pathway also seems to be activated by certain beneficial bacteria, suggesting that different beneficial and pathogenic microbes initiate common plant signaling pathways.

The Role of Plant Growth Promoting Bacteria. PGPB belong to a number of different bacterial families, including Rhizobium, Bacillus, Pseudomonas and Burkholderia. PGPB can improve plant growth under abiotic stress conditions. Enhanced salt tolerance of Zea mays upon co-inoculation with Rhizobium and Pseudomonas is correlated with decreased electrolyte leakage and maintenance of leaf water contents. Some microbes produce plant hormones, such as indole acetic acid and gibberellic acid, which induce increased root growth and thereby lead to enhanced uptake of nutrients.

Plants have the ability to acquire a state of induced systemic resistance (see Chap. 13) to pathogens after inoculation with PGPB. In association with plant roots, PGPB can prime the plant's innate immune system and confer resistance to a broad spectrum of pathogens with a minimal impact on yield and growth. Several PGPB colonize roots and protect a large variety of plant species, including vegetables, crops and even trees, against foliar diseases in greenhouse and field trials. 
Plant-Associated Fungi Confer Stress Tolerance to Plants. Mycorrhizal and/or endophytic fungi interact with many plant species and significantly contribute to the adaptation of these plants to a number of environmental stresses including drought, heat, pathogens, herbivores or limiting nutrients. Some plants are unable to withstand stress conditions in the absence of their associated microbes. It appears that stress tolerance of the host plant can be a habitatspecific feature of the interaction. For example, Curvularia protuberata confers heat tolerance to its geothermal host plant Dichanthelium lanuginosum. However, neither the fungus nor the plant can survive alone at temperatures above $38^{\circ} \mathrm{C}$ (Redman et al. 2002). Moreover, only $C$. protuberata isolates from geothermal plants can confer heat tolerance (Rodriguez et al. 2008). A comparison of different fungal endophytes unravels a further layer of specificity: $C$. protuberata confers heat but neither disease nor salt tolerance. In contrast, Fusarium culmorum only confers salt tolerance and Curvularia magna only disease tolerance. It appears that these specific features contribute to the ability of some plants to establish and survive in extreme habitats.

Symbiotically conferred disease tolerance appears to involve different mechanisms, depending on the endophyte. For example, a non-pathogenic Colletotrichum strain that confers disease resistance does not activate host defense in the absence of pathogen challenge (Redman et al. 1999). Moreover, disease resistance is localized to tissues that the fungus has colonized, but is not systemic.

In contrast, Piriformospora indica confers disease resistance systemically. $P$. indica colonizes the roots of many plant species and stimulates growth, biomass and seed production. $P$. indica promotes nitrate and phosphate uptake and confers resistance against abiotic and biotic stress (Waller et al. 2005). The fungal colonization stimulates the host to synthesize phosphatidic acid, which triggers the OXI1 pathway (Camehl et al. 2011). This pathway is usually activated only in response to pathogen attack to activate host defense. A defect in the OXI1 pathway negatively affects plant growth by the fungus, resembling a pathogenic interaction. Overall, the differences between Colletotrichum spp.- and P. indicaconferred disease resistance indicate a number of different mechanisms yet to be elucidated.

Further evidence indicates that our present concepts of categorizing microbes as pathogenic or beneficial are inadequate. For example, Fusarium culmorum can cause disease on a variety of crop plants. However, the F. culmorum isolate FcRed1 is beneficial and confers salt tolerance to its host dunegrass Leymus mollis, but isolates from non-coastal dunegrass do not. C. protuberata is a plant pathogen for several monocots, but isolate Cp4666D confers heat and drought tolerance to its host plant Dichanthelium lanuginosum. While Curvularia species are not known to have broad disease-host ranges, C. protuberata from the monocot $D$. lanuginosum also confers heat tolerance on tomato (Márquez et al. 2007; Rodriguez et al. 2008). Some microbes can also be present in plants without showing disease symptoms. For example, Colletotrichum acutatum can colonize pepper, eggplant, bean and tomato without causing disease, but with other plants, such as strawberry, disease symptoms become evident (Freeman et al. 2001). So it appears that a number of microbes have a host-dependent lifestyle as pathogenic or beneficiary partner of plants, but the molecular basis of the plant-microbe interactions remains to be unraveled. 


\subsection{Desert Farming Exploits Plant-Microbe Interaction to Improve Water Management}

Beneficial plant-associated microbes can help plants suppress diseases, stimulate growth, occupy space that would otherwise be taken up by pathogens, promote biotic stress resistance, and increase crop yield and quality by nutrient mobilization and transport (Berg et al. 2013). Therefore, the plant microbiome is a key determinant of plant health and productivity. While the possibility to control biotic stress by plant-associated microorganisms is known since more than 100 years, less is known about controlling abiotic stress. There are many biocontrol products on the market but our understanding how plant-associated microbes can compensate abiotic stress is only at the beginning. However, several promising examples are already reported in the literature. Armada et al. (2014) showed that strains of Bacillus megaterium, Enterobacter sp., Bacillus thuringiensis, and Bacillus sp. have the potential to alleviate drought stress in Lavandula and Salvia by increasing K content, by depressing stomatal conductance, or by controlled shoot proline accumulation. The production of osmoprotectans was also identified as a key mechanism of the Stress Protecting Agent Stenotrophomonas rhizophila DSM14405T (Alavi et al. 2013). In addition, this strain produced spermidine, which is a general, highly efficient stress protectant. In another example, pepper plants exposed to bacterial isolates from plants cultivated under desert farming exhibited a higher tolerance to water shortage, compared with untreated control (Marasco et al. 2012; Marasco et al. 2013b). This promotion was mediated by a larger root system (up to $40 \%$ ), stimulated by the bacteria that enhanced the plant's ability to take up water from dry soil. Altogether, to exploit plant-microbe interaction to improve water management in desert farming is a challenging but also promising task for the future.

\section{References}

Adam M, Heuer H, Hallmann J (2014) Bacterial antagonists of fungal pathogens also control root-knot nematodes by induced systemic resistance of tomato plants. PLoS One 9:e90402.

Alavi P, Starcher MR, Zachow C et al (2013) Root-microbe systems: the effect and mode of interaction of Stress Protecting Agent (SPA) Stenotrophomonas rhizophila DSM14405 ${ }^{\mathrm{T}}$. Front Plant Sci 4:141

Angel R, Soares MI, Ungar ED et al (2010) Biogeography of soil archaea and bacteria along a steep precipitation gradient. ISME J 4:553-563

Apel K, Hirt H (2004). Reactive oxygen species: metabolism, oxidative stress, and signal transduction. Ann Rev Plant Biol 55:373-399

Armada E, Roldán A, Azcon R (2014) Differential activity of autochthonous bacteria in controlling drought stress in native lavandula and salvia plants species under drought conditions in natural arid soil. Microb Ecol $67: 410-420$

Bartels D, Sunkar R (2005) Drought and salt tolerance in plants. Critical Rev Plant Sci 24:23-25 
Berg G, Zachow C, Müller H et al (2013) Next-generation bio-products sowing the seeds of success for sustainable agriculture. Agronomy 3:648-656

Camehl I, Drzewiecki C, Vadassery Y et al (2011) The OXI1 kinase pathway mediates Piriformospora indicainduced growth promotion in Arabidopsis. PloS Pathog 7:e1002051

Cho SM, Kang BR, Han SH et al (2008) 2R,3R-butanediol, a bacterial volatile produced by Pseudomonas chlororaphis 06, is involved in induction of systemic tolerance to drought in Arabidopsis thaliana. Mol PlantMicrobe Int 21:1067-1075

Clery D (2011) Environmental technology. Greenhouse-power plant hybrid set to make Jordan's desert bloom. Science 331:136

Corradi N, Bonfante P (2012) The arbuscular mycorrhizal symbiosis: origin and evolution of a beneficial plant infection. PLoS Pathog 8:e1002600

De Los Ríos A, Valea S, Ascaso C et al (2010) Comparative analysis of the microbial communities inhabiting halite evaporites of the Atacama Desert. Int Microbiol 13:79-89

de Zelicourt A, Al-Yousif M, Hirt H (2013) Rhizosphere microbes as essential partners for plant stress tolerance. Mol Plant 6:242-245

Ding GC, Piceno YM, Heuer H et al (2013) Changes of soil bacterial diversity as a consequence of agricultural land use in a semi-arid ecosystem. PLoS One 8:e59497

Fierer N, Jackson RB (2006) The diversity and biogeography of soil bacterial communities. Proc Natl Acad Sci USA 103:626-631

Freeman S, Horowitz S, Sharon A (2001) Pathogenic and non-pathogenic lifestyles in Colletotrichum acutatum from strawberry and other plants. Phytopathology 91:986-99.

Geurts R, Lillo A, Bisseling T (2012) Exploiting an ancient signalling machinery to enjoy a nitrogen fixing symbiosis. Curr Opin Plant Biol 15:438-443

Hasegawa S, Meguro A, Nishimura T, Kunoh H (2004). Drought tolerance of tissue-cultured seedlings of mountain laurel (Kalmia latifolia L.) induced by an endophytic actinomycete. I. Enhancement of osmotic pressure in leaf cells. Actinomycetology, 18:43-47

Heidari M, Mousavinik SM, Golpayegani A (2011) Plant growth promoting rhizobacteria (PGPR) effct on physiological paramters and mineral uptake in basil (Ociumum basilicum L.) under water stress. ARPN J Agric Biol Sci 6:6-11

Köberl M, Müller H, Ramadan EM et al (2011) Desert farming benefits from microbial potential in arid soils and promotes diversity and plant health. PloS ONE. 6:e24452

Köberl M, Ramadan EM, Adam M et al (2013a) Bacillus and Streptomyces were selected as broad-spectrum antagonists against soilborne pathogens from arid areas in Egypt. FEMS Microbiol Lett 342:168-178

Köberl M, Schmidt R, Ramadan EM et al (2013b) Biocontrol strategies and next generation sequencing: organic desert agriculture in Egypt. iConcept, online

Kohlera J, Hernández JA, Caravacaa A et al (2009) Induction of antioxidant enzymes is involved in the greater effectiveness of a PGPR versus AM fungi with respect to increasing the tolerance of lettuce to severe salt stress. J Exp Bot 65:245-252

Marasco R, Rolli E, Ettoumi B et al (2012) A drought resistance-promoting microbiome is selected by root system under desert farming. PLoS One 7:e48479 
Marasco R, Rolli E, Fusi M et al (2013a) Plant growth gromotion potential is equally represented in diverse grapevine root-associated bacterial communities from different biopedoclimatic environments. BioMed Res Int 2013:491091

Marasco R, Rolli E, Vigani G et al (2013b) Are drought-resistance promoting bacteria cross-compatible with different plant models? Plant Signal Behav 10:e26741

Márquez LM, Redman RS, Rodriguez RJ et al (2007) A virus in a fungus in a plant-three way symbiosis required for thermal tolerance. Science 315:513-515

Mayak S, Tirosh T, Glick B (2004) Plant growth-promoting bacteria that confer resistance to water stress in tomatoes and peppers. Plant Sci 166:525-530

Park S, Li J, Pittman JK et al (2005) Up-regulation of a $\mathrm{H}^{+}$-pyrophosphatase ( $\mathrm{H}^{+}$-PPase) as a strategy to engineer drought-resistant crop plants. Proc Natl Acad Sci USA 102:18830-18835

Redman RS, Freeman S, Clifton DR et al (1999) Biochemical analysis of plant protection afforded by a nonpathogenic endophytic mutant of Colletotrichum magna. Plant Physiol 119:795-804

Redman RS, Sheehan KB, Stout RG et al (2002) Thermotolerance conferred to plant host and fungal endophyte during mutualistic symbiosis. Science 298:1581

Rodríguez-Salazar R, Suárez R, Caballero-Mellado J et al (2009) Trehalose accumulation in Azospirillum brasilense improves drought tolerance and biomass in maize plants. FEMS Microbiol Lett 296:52-59

Rodriguez RJ, Henson J, Van Volkenburgh E et al (2008) Stress tolerance in plants via habitat-adapted symbiosis. ISME J 2:404-416

Rolli E, Marasco M, Vigani G et al (2014) Improved plant resistance to drought is promoted by the rootassociated microbiome as a water stress-dependent trait. Environ Microbiol doi:10.1111/1462-2920.12439

Schmidt R, Köberl M, Mostafa A et al (2014) Effects of bacterial inoculants on the indigenous microbiome and secondary metabolites of chamomile plants. Front Microbiol 5:64

Waller F, Achatz B, Baltruschat H et al (2005) The endophytic fungus Piriformospora indica reprograms barley to salt-stress tolerance, disease resistance, and higher yield. Proc Natl Acad Sci USA 102:13386-13391

\section{Additional info:}

a. Estimated number of pages: 11.98

b. Name and address (including full postal address) of corresponding author:

Daniele Daffonchio, Department of Food, Environmental and Nutritional Sciences

(DeFENS), University of Milan, I-20133 Milan, Italy \& King Abdullah University of Science and Technology, BESE Division, Thuwal, 23955-6900, Kingdom of Saudi Arabia

Email: daniele.daffonchio@unimi.it; mobile +393339742943

c. Abbreviations: 
ABA abscisic acid; ACC, 1-aminocyclopropane-1-carboxylic acid; EPS, exopolymers; GAs, gibberellins; PGP, Plant Growth Promoting; PGPB, Plant Growth Promoting Bacteria; PGPF, Plant Growth Promoting Fungi; ROS, Reactive Oxygen Species; VOCs, Volatile Organic Compounds;

d. Entries for INDEX:

Drought

Desert Farming

Plant Growth Promoting Bacteria

e. No Figures included

f. No Tables included

g. USA English

h. MS Checklist: everything in order

i. Forms submitted together with the MS:

- Consent to Publish

j. I would like to put the following additional material on SpringerLink: none 